\title{
Room-temperature synthesis of gold nanoparticles - Size-control by slow addition
}

\author{
Sankar K. Sivaraman, Sanjeev Kumar and Venugopal Santhanam* \\ www.goldbulletin.org
}

\begin{abstract}
We report a simple and rapid process for the roomtemperature synthesis of gold nanoparticles using tannic acid, a green reagent, as both the reducing and stabilising agent. We systematically investigated the effect of $\mathrm{pH}$ on the size distribution of nanoparticles synthesized. Based on induction time and $\zeta$-potential measurements, we show that particle size distribution is controlled by a fine balance between the rates of reduction (determined by the initial $\mathrm{pH}$ of reactants) and coalescence (determined by the $\mathrm{pH}$ of the reaction mixture) in the initial period of growth. This insight led to the optimal batch process for size-controlled synthesis of 2-10 $\mathrm{nm}$ gold nanoparticles - slow addition (within 10 minutes) of chloroauric acid into tannic acid.
\end{abstract}

Department of Chemical Engineering, Indian Institute of Science, Bangalore, India 560012.

\footnotetext{
${ }^{*}$ Corresponding author

Email:venu@chemeng.iisc.ernet.in
}

Keywords: gold nanoparticles, room-temperature, size-control, slow addition, tannic acid

\section{Introduction}

Gold nanoparticles in the 1-10 $\mathrm{nm}$ size range have size-tuneable physico-chemical properties $(1,2)$ that are useful in a wide variety of applications such as cancer diagnostics (3), catalysis (4), Raman and fluorescence spectroscopy $(5,6)$, selective ionisation of biomolecules (7), and single electronics $(8,9)$. Commercial realisation of such applications relies on economical synthesis of size-controlled nanoparticles, which requires the development of continuous-flow versions of lab-scale processes. Among the various lab-scale processes, solutionphase redox reactions are currently in wide use for synthesizing gold nanoparticles in the size range of 1-10 $\mathrm{nm}$, due to their relative energy-efficiency and ease of implementation (10).

Central to the development and success of solutionphase processes for size-controlled continuous, nanoparticle synthesis are issues related to economic and environmental viability (11). Resolution of these issues warrants the development of lab-scale processes having fast process completion times, using green reagents in aqueous phase at ambient conditions. The use of various naturally available reagents as reducing agents for aqueous-phase redox synthesis of colloidal gold is well documented by Weiser $(12)$; recent reports $(13,14)$ exemplify the growing library of such reagents. Presently, most green processes either offer moderate control over polydispersity or require significant thermal energy input (on account of the high specific heat capacity of water, $\sim 4.186 \mathrm{~J} /[\mathrm{g} . \mathrm{K}]$ ) to realize reasonable batch processing times, hindering their adaptation for continuous flow processing. Typically, the use of hazardous reducing agents (e.g. sodium borohydride, hydrazine) in conjunction with other stabilisers is used to achieve rapid, roomtemperature synthesis of size-controlled gold nanoparticles $(15,16)$. 
Recently (17), we demonstrated room temperature synthesis of silver nanoparticles in seconds using tannic acid at alkaline $\mathrm{pH}$ as the reducing and stabilising agent. Tannic acid, a polyphenolic compound derived from plants, has been used as a reducing agent and a protective colloid in gold nanoparticle synthesis for a long time. Ostwald (18) reported that tannin can reduce chloroauric acid at neutral $\mathrm{pH}$ to form stable gold nanoparticles even if tap water is used to prepare the aqueous solutions. Tannic acid is also an important constituent of the widely used Slot and Geuze protocol (19) for synthesizing gold nanoparticles in the size range of 3-17 nm with nominal standard deviation $(\sigma)$ of 0.5$1.8 \mathrm{~nm}(20)$ at $60^{\circ} \mathrm{C}$. Turkevich et al. (21) replicated Ostwald's protocol and reported the particle size to be $12.0 \pm 3.6 \mathrm{~nm}(\mu \pm \sigma)$ based on TEM images. The objective of the present work was to develop an optimal batch process using tannic acid, for the rapid, size-controlled synthesis of 1-10 nm gold nanoparticles at ambient conditions.

In the following, we show the effect of varying the initial molar ratio of tannic acid to chloroauric acid (MR) on the particle size distribution and morphology. This is followed by a systematic investigation of the effect of $\mathrm{pH}$ on nanoparticle formation rate and coalescence, as characterised by TEM, induction time, and $\zeta$-potential measurements. Next, we show the effect of varying the mode of contacting the reagents, and mixing on nanoparticle size distribution. These studies revealed the importance of the dynamics of transformation among various chloroauric acid species in solution, and the competition between coalescence and stabilisation during the initial period of nanoparticle formation. Such insights led us to the optimal batch process for size-controlled synthesis: slow (dropwise) addition of chloroauric acid into tannic acid, which is contrary to prevalent practice (22-24). This process has the potential to be extended to continuous mode of synthesis with residence time of the order of minutes.

\section{Experimental methods}

\subsection{Materials}

Hydrogen tetrachloroaurate (III) hydrate (99.999\%) and tannic acid (ACS reagent) were purchased from Sigma-Aldrich Co. The replicate experiments reported in Figure 7 were carried out using chemicals of similar purity purchased from Acros organics Ltd. Potassium carbonate was purchased from Merck Co. Deionized water obtained from a MilliQ ${ }^{\circledR}$ system was used in all the experiments. All chemicals were used as received.

\subsection{Synthesis of gold nanoparticles}

The experiments were carried out in glassware cleaned with aquaregia (CAUTION: Aquaregia solutions are extremely corrosive), rinsed thoroughly with deionized water and then dried in air prior to use. For the preliminary investigations and experiments 'A' - ' $F$ ', the precursor solutions were prepared as follows. $0.25 \mathrm{~mL}$ of $25.4 \mathrm{mM}$ chloroauric acid solution was made up to $22 \mathrm{~mL}$ using DI water. $3 \mathrm{~mL}$ of the desired concentration of tannic acid solution was prepared. $150 \mathrm{mM}$ potassium carbonate solution was used to adjust the $\mathrm{pH}$ of tannic acid and/or chloroauric acid solution. If necessary, 0.1 $\mathrm{N}$ hydrochloric acid was used to adjust the $\mathrm{pH}$ of chloroauric solution. A reactor equipped with homemade Rushton turbine and baffles of standardized design (25) machined out of Teflon was used to mix the reactants (macroscopic blending time of 2s). Typically, chloroauric acid solution was taken in the stirred vessel and the reducing agent was added as one portion (within 1 second). The reaction mixture was stirred till the colour of the colloidal solution did not appear to change.

For Stop Flow Module (SFM) studies (experiment ' $H$ '), stock solutions of chloroauric acid and tannic acid were prepared as follows. Briefly, $0.25 \mathrm{~mL}$ of $25.4 \mathrm{mM}$ chloroauric acid was made up to $20 \mathrm{~mL}$ using DI water. $2.25 \mathrm{~mL}$ of $5.9 \mathrm{mM}$ tannic acid was made up to $5 \mathrm{~mL}$ using DI water.

Experiment 'l' was carried out by adding $10 \mathrm{~mL}$ of $0.64 \mathrm{mM}$ chloroauric acid solution ( $\mathrm{pH}-3.2)$ slowly (dropwise, $\sim 1 \mathrm{~mL} / \mathrm{min}$ ) into $15 \mathrm{~mL}$ of $0.89 \mathrm{mM}$ tannic acid solution $(\mathrm{pH}-7.1)$ that was being stirred. The total volumes and amounts of reagents used were kept at the same value as experiment ' $B$ ' and ' $G$ '. For seeded growth experiment reported in Figure 6f, 2.5 $\mathrm{mL}$ (from a total of $25 \mathrm{~mL}$ ) of the solution formed in experiment 'l' was made upto $15 \mathrm{~mL}$ using DI water and $10 \mathrm{~mL}$ of $0.32 \mathrm{mM}$ chloroauric acid solution was added slowly (dropwise) into it. The $\mathrm{pH}$ was adjusted to be above 6.4 by intermittently adding required quantities of $150 \mathrm{mM}$ potassium carbonate solution.

\subsection{Sample characterisation}

Transmission Electron Microscopy (TEM) characterisation was performed using a Tecnai F30 operating at 200 KV. Samples for TEM were prepared by drop casting and ImageJ (http://rsbweb.nih.gov/ ij) software was used for analysing TEM images. 
The mean $\left(\mu \equiv \Sigma \mathrm{d}_{\mathrm{i}} / \mathrm{n}\right)$ and standard deviation $\left(\sigma \equiv \sqrt{ }\left(\Sigma\left(d_{i}-\mu\right)^{2} /(n-1)\right)\right.$ of the nanoparticle size distribution are reported along with the size histograms displaying the actual number of particles counted.

UV-Vis spectra were collected using a Systronics double beam UV visible spectrophotometer 2201. DI water was used as the reference. Stop flow reactor (SFM-400, Biologic SA, Claix, France) equipped with xenon lamp and photomultiplier tube was used to monitor, with $10 \mu$ s time resolution, the absorbance of the reaction mixture at the desired wavelength after rapid mixing (dead time $\sim 3 \mathrm{~ms}$ ) of the reactants.

The \% yield of the synthesis was measured by carrying out Atomic Absorption Spectrometry of gold precipitate using GBC Avanta 3000 (graphite furnace). Standard curve method was used for calibration and analysis. For sample preparation, $5 \mathrm{~mL}$ of colloidal gold solution was precipitated by the addition of $5 \mathrm{~mL}$ of ethanol and $3 \mu \mathrm{L}$ of dodecanethiol followed by centrifugation at $3000 \mathrm{~g}$ for $30 \mathrm{~min}$. The nanoparticle precipitate was then dissolved in $5 \mathrm{~mL}$ of aquaregia.

The average hydrodynamic particle size $\left(d_{h}\right)$ and standard deviation were measured, as per ISO 13321, at $90^{\circ}$ scattering angle using a Dynamic Light Scattering (DLS) instrument (Model BI-200SM) from Brookhaven Co. equipped with BI-9000AT correlator system. It was observed that the hydrodynamic size obtained from DLS was always greater than TEM mean diameter indicating the presence of an adsorbed layer, and the variability was very sensitive to the presence of non-spherical particles.

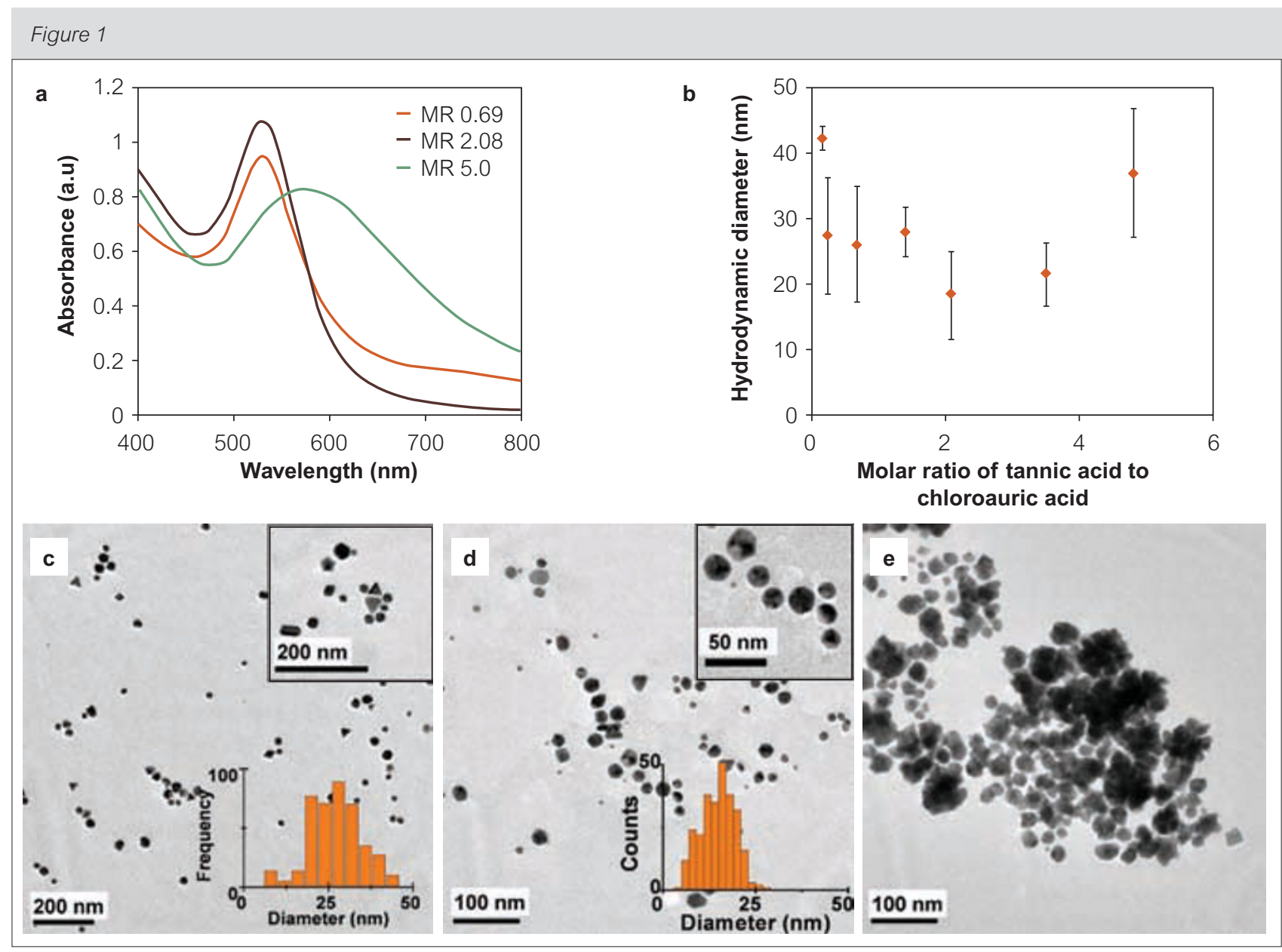

Effect of varying the initial molar ratio of tannic acid to chloroauric acid (MR). (a) UV visible spectra of colloids synthesized at different $M R$ values. A red shift is observed in the surface plasmon peak beyond MR value of 2.08. (b) Variation of hydrodynamic diameter with MR. The error bars represent the standard deviation of the estimates of mean size obtained from six repeated measurements. (c) - (e) Representative TEM image of nanoparticles synthesized at different MR values. Insets show higher magnification images and size distribution histograms. (c), MR 0.69. Nanoparticle diameter $(\mu \pm \sigma)=25.3 \pm 8.0 \mathrm{~nm}$. (d) MR 2.08. Nanoparticle diameter $(\mu \pm \sigma)=14.1$ $\pm 4.8 \mathrm{~nm}$. (e) MR 5.2 
$\zeta$-potential values of gold colloidal solutions were measured either using a ZetaPlus analyzer from Brookhaven instruments or a Malvern Zetasizer, and the mean and standard deviation values based on three repeated measurements are reported. $\mathrm{pH}$ measurements were made using Orion ${ }^{\mathrm{TM}} \mathrm{pH}$ electrode and benchtop meter.

The redox potentials of freshly prepared $0.53 \mathrm{mM}$ tannic acid solution (corresponding to MR 2.08) at different $\mathrm{pH}$ were measured using a saturated calomel electrode (SCE) as reference and platinum wire as the working electrode. The values reported are with respect to SCE, and were measured after allowing sufficient time to equilibrate.

\section{Results and discussion}

\subsection{Variation of mean nanoparticle size with MR value}

Slot and Geuze (19) synthesized gold nanoparticles of varying size, ranging from $12 \mathrm{~nm}$ to $3.5 \mathrm{~nm}$, simply by increasing the molar ratio of tannic acid to chloroauric acid in the presence of sodium citrate. Although this protocol has been widely used in the last three decades, there is no report on the use of higher amounts of tannic acid to form nanoparticles of even smaller sizes. During preliminary experiments, aimed at understanding this aspect, we noticed that the addition of only tannic acid in amounts higher than the maximum value reported by Slot and Geuze resulted in the formation of gold sols even at room temperature $\left(24-28^{\circ} \mathrm{C}\right)$, on a timescale of minutes. So, we synthesized gold colloids at various $\mathrm{MR}$ values without altering the natural $\mathrm{pH}$ of the solutions. All the MR values chosen were greater than the stoichiometric requirement (17) for complete reduction of gold salt. A few experiments carried out at different overall concentrations of gold chloride and tannic acid (varied from 25\% to 200\% of the standard value used) also yielded the same final nanoparticle size distribution as the samples reported herein for the corresponding MR values.

Figure 1a shows the variation of UV-Vis spectra as a function of the MR value. The surface plasmon resonance (SPR) peak in the UV-Vis spectra of gold nanoparticle solutions formed at MR values higher than 2.08 were red shifted with respect to those formed at lower MR values, indicating the presence of large particles, while those formed at MR values below 2.08 exhibit increased absorbance in the NIR region indicating the presence of anisotropic and faceted particles. Figure $1 \mathrm{~b}$ shows the variation of hydrodynamic diameter with MR; the trend is in accord with that observed in UV-Vis spectra, with a minimum at MR value of 2.08. Representative TEM images of a few of these samples are also shown in figures 1c-1e, and the trend in the mean size is in agreement with UV-Vis and DLS data.

The TEM image of the sample prepared at an MR value of 5.2 has several branched flower-like particles that are similar to the 'nanoroses' described in a recent report (26). The mean size (diameter of an equivalent circle having the same projected area) is not reported due to the highly irregular shape of the particles. The TEM image of the sample prepared at MR value of 0.69 shows that many particles are highly faceted with triangular or bipyramidal shapes, while the sample prepared at MR value of 2.08 shows predominantly quasispherical particles and a smaller fraction of particles with triangular/bipyramidal shapes. AAS analysis of the sample prepared with an MR 2.08 indicated that the yield was $90.8 \pm 5.6 \%$ (95\% confidence limits), and that there was no gold remaining in the supernatant (within detectable limits, i.e. $>3.07 \%$ ). This proves the quantitative conversion of gold present as ions in the precursor into atoms constituting the nanoparticles. The nonmonotonic variation with MR of the size and shape of nanoparticles is currently under investigation as simple arguments based on increased reactivity and stabilisation due to an increase in tannic acid concentration cannot explain the minima. As the objective was to synthesize spherical gold nanoparticles rapidly, further experiments were all carried out at an overall MR value of 2.08.

\subsection{Role of $\mathrm{pH}$}

Ostwald (18) cautioned that the $\mathrm{pH}$ of the chloroauric acid precursor solution had to be near neutral to ensure the stability (based on formation of a ruby coloured solution) of the resultant dispersion formed by reduction with tannin at its natural $\mathrm{pH}$, which is acidic. Typically, changes in gold nanoparticle size or shape distributions resulting from a change in $\mathrm{pH}$ of the reaction mixture are ascribed to either a variation in the reactivity of the precursors and/or the strength of stabilisation of nanoparticles (19, 27-30). As the $\mathrm{pH}$ values required for maximising precursor reactivity and nanoparticle stabilisation differ significantly, we decided to explore conditions under which both reactivity and stabilisation could be optimised. So, we carried out a series of experiments under ambient conditions, by varying the initial $\mathrm{pH}$ of the two precursor solutions and characterised the resulting colloidal samples using TEM, induction 


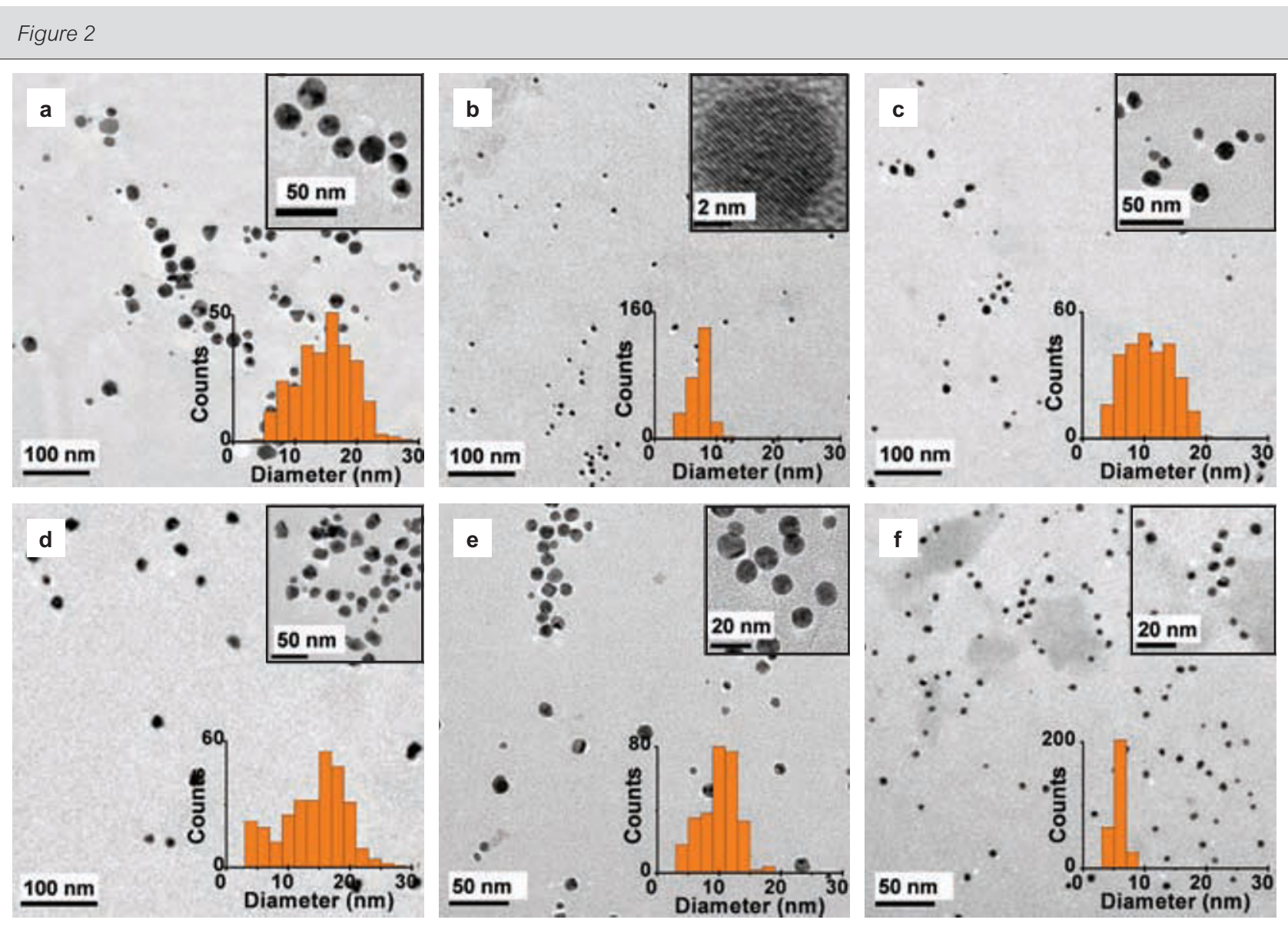

Representative TEM images of nanoparticles synthesized by varying reactant $\mathrm{pH}$. Insets show higher magnification images and size distribution histograms. (a) Tannic acid at pH 3.1 was added to chloroauric acid at pH 3.2. Nanoparticle diameter $(\mu \pm \sigma)=14.1 \pm 4.8$ nm. (b) Tannic acid at pH 7.1 was added to chloroauric acid at pH 3.2. Nanoparticle diameter $(\mu \pm \sigma)=7.1 \pm 1.6 \mathrm{~nm}$. (c) Tannic acid at pH 6.3 was added to chloroauric acid at pH 7.0. Nanoparticle diameter $(\mu \pm \sigma)=10.7 \pm 3.8 \mathrm{~nm}$. (d) Tannic acid at pH 3.1 was added to chloroauric acid at pH 9.1. Nanoparticle diameter $(\mu \pm \sigma)=14.2 \pm 5.4 \mathrm{~nm}$. (e) Tannic acid at pH 7.1 was added to chloroauric acid at pH 2.1. Nanoparticle diameter $(\mu \pm \sigma)=10.0 \pm 2.9 \mathrm{~nm}$. (f) Tannic acid at pH 9.0 was added to chloroauric acid at pH 2.1. Nanoparticle diameter $(\mu \pm \sigma)=5.8 \pm 1.0 \mathrm{~nm}$

time, and $\zeta$-potential measurements. In the following, we focus our discussion on six experiments (labelled ' $A$ ' - ' $F$ '), which provide an insight into the various processes modulated by changes in $\mathrm{pH}$ that affect the observed particle size distribution. The experimental conditions used, and the results of sample characterisations are presented in Table 1. Representative TEM images of these samples are shown in Figure 2. The measured lattice spacing $(0.232 \pm 0.008 \mathrm{~nm})$ of the fringes seen in the inset of figure $2 \mathrm{~b}$ corresponds to the value expected for gold (111).

Experimental runs ' $A$ ' and ' $B$ ' were carried out to investigate the effect of varying the reactivity of tannic acid on nanoparticle synthesis. It is seen that both the nanoparticle mean diameter and polydispersity reduces upon an increase in tannic acid $\mathrm{pH}$. Since the reduction in induction time (a measure of reactivity) is moderate while the $\zeta$-potential changes significantly from $-20 \mathrm{mV}$ to $-49 \mathrm{mV}$, the observed decrease in mean size and polydispersity is mainly attributed to a decrease in coalescence caused by increased strength of stabilisation in experiment 'B'. Given that both these samples were stable upon storage for more than a year, coalescence is inferred to occur mainly during the initial period of nanoparticle growth. This supposition, i.e. that the value of $\zeta$-potential measured in experiment A $(-20 \mathrm{mV})$ is below the threshold for stabilising small $(<2 \mathrm{~nm})$ nanoparticles, is in accord with earlier reports (31).

To study the effect of precursor reactivity on nanoparticle synthesis, experiments ' $C$ ' and 'D' were conducted by varying the $\mathrm{pH}$ of reagents while 


\begin{tabular}{|c|c|c|c|c|c|c|}
\hline Label & $\begin{array}{l}\text { Initial pH of } \\
\text { chloroauric } \\
\text { acid solution }\end{array}$ & $\begin{array}{l}\text { Initial pH of } \\
\text { tannic acid } \\
\text { solution }\end{array}$ & $\begin{array}{l}\mathrm{pH} \text { of reaction } \\
\text { mixture }\end{array}$ & $\begin{array}{l}\text { Particle diameter }^{\mathrm{a}} \\
(\mu \pm \sigma), \mathrm{nm}\end{array}$ & $\begin{array}{l}\text { Induction } \\
\text { time }^{b}, s\end{array}$ & $\begin{array}{l}\zeta \text {-potentialc, } \\
\mathrm{mV}\end{array}$ \\
\hline$A$ & 3.2 & 3.1 & 3.2 & $14.1 \pm 4.8$ & 3 & $-20 \pm 2$ \\
\hline$B$ & 3.2 & 7.1 & 6.4 & $7.1 \pm 1.6$ & 1.5 & $-49 \pm 2$ \\
\hline C & 7.0 & 6.3 & 6.4 & $10.7 \pm 3.8$ & 66.8 & $-69 \pm 2$ \\
\hline$D$ & 9.1 & 3.1 & 6.4 & $14.2 \pm 5.4$ & 163.5 & $-60 \pm 2$ \\
\hline$E$ & 2.1 & 7.1 & 5.0 & $10.0 \pm 2.9$ & 0.9 & $-18 \pm 7$ \\
\hline $\mathrm{F}$ & 2.1 & 9.0 & 7.1 & $5.8 \pm 1.0$ & 0.5 & $-35 \pm 7$ \\
\hline
\end{tabular}

ensuring that the resultant reaction mixture $\mathrm{pH}$ was the same as in experiment ' $\mathrm{B}$ '. It is observed that the nanoparticle size and polydispersity increase with an increase in the initial $\mathrm{pH}$ of the chloroauric acid solution. The $\zeta$-potential values measured in experiments 'B', 'C', and 'D' are sufficiently above the threshold value to preclude coalescence as the major factor responsible for the observed changes in nanoparticle size distribution amongst these three cases. The large increase in induction time clearly indicates that it is the decrease in chloroauric acid reactivity at higher $\mathrm{pH}$ values (28) that results in small nucleation rates and prolonged nucleation periods (as nucleation will continue till sufficient nanoparticle surface area is generated for growth to dominate), thereby accounting for the observed changes in nanoparticle size distribution at the same overall reaction mixture $\mathrm{pH}$. However, this deduction presumes that the reactivity of chloroauric acid is dependent on its initial $\mathrm{pH}$ and not on the reaction mixture $\mathrm{pH}$.

It is reported that chloroauric acid is present mainly as a combination of gold chloro-hydroxy species in aqueous solutions, and that the reactivity decreases as the chloride ligands are displaced by hydroxyl ligands at higher $\mathrm{pH}$ values $(28,30)$. It is also known that the absorbance at $315 \mathrm{~nm}$ corresponding to the charge transfer to solvent (CTTS) absorption band depends on the number of chloride ligands

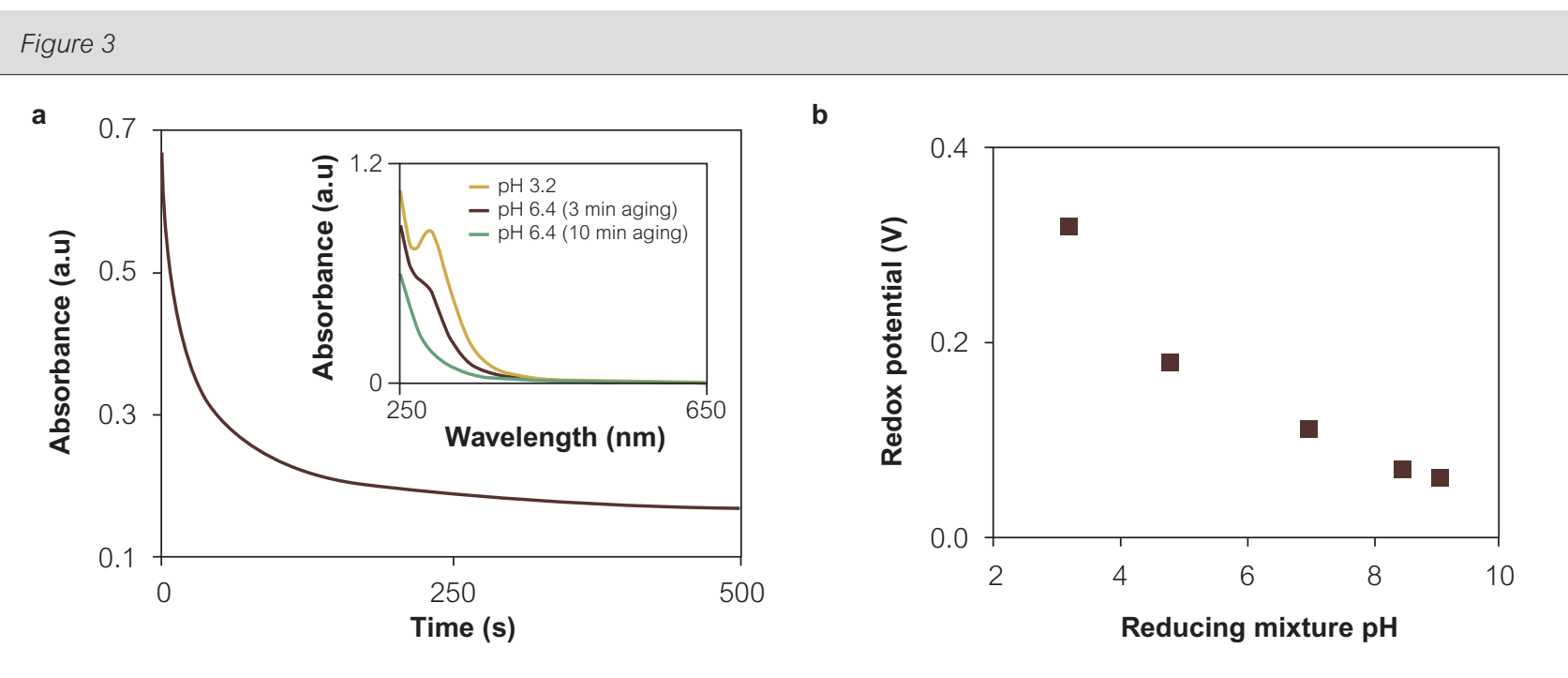

(a) The dynamics of absorbance at $315 \mathrm{~nm}$ monitored using an SFM at ms resolution, after rapidly mixing chloroauric acid solution at $\mathrm{pH} 3.2$ with $\mathrm{K}_{2} \mathrm{CO}_{3}$ solution to change the $\mathrm{pH}$ to 6.4. The inset shows UV-Vis spectra (measured ex-situ) of a similar solution at various time intervals after increasing the $\mathrm{pH}$ from 3.2 to 6.4. (b) Redox potential (w.r.t. SCE) of tannic acid at different pH 
present (32). So, SFM experiments were conducted to study the effect of a sudden change in $\mathrm{pH}$ on the transformation of chloroauric acid species from one form into another, by monitoring the CTTS band of gold chloride at $315 \mathrm{~nm}$. Figure 3 a shows that the response time for chloroauric acid species transformation is of the order of 3-4 minutes, for a change in $\mathrm{pH}$ from 3.2 to 6.4. As this timescale is larger than the timescale for completion of reaction in experiment $\mathrm{B}$, it indeed establishes that the reactivity of chloroauric acid solution is controlled by its initial $\mathrm{pH}$ and not the reaction mixture $\mathrm{pH}$.

To delineate the effect of the reaction mixture $\mathrm{pH}$ from that of precursor reactivity, experiments 'E' and 'F' were conducted with chloroauric acid maintained at $\mathrm{pH} 2.1$ and tannic acid at $\mathrm{pH} 7.1$ and 9, this resulted in final reaction mixture $\mathrm{pH}$ values of 5.0 and 7.1 respectively. The redox potential of tannic acid increases only slightly over this range (Fig. 3b), while chloroauric acid is expected to be in a more reactive state than in experiment 'B'. The measured values of induction time ( 0.9 and $0.5 \mathrm{~s})$ are in agreement with these expectations. However, the observed value of nanoparticle size distribution $(10.0 \pm 2.9 \mathrm{~nm})$ in experiment ' $\mathrm{E}$ ' is larger than that in experiment ' $\mathrm{B}$ ' $(7.1 \pm 1.6 \mathrm{~nm})$, while that observed in experiment ' $F$ ' $(5.8 \pm 1.0 \mathrm{~nm})$ is smaller. The $\zeta$-potential values measured in experiments ' $E$ ' and ' $F$ ' (-18 and $-35 \mathrm{mV}$ ) clearly indicate that the strength of stabilisation has substantially decreased in the case of experiment 'E', signifying that coalescence was the reason for the observed larger size and polydispersity.

Comparing the data from experiments 'A', 'B', ' $E$ ' and ' $F$ ', it is clear that the strength of stabilisation (as characterized by the magnitude of $\zeta$-potential) is a function of reaction mixture $\mathrm{pH}$ and it changes considerably over a narrow range of $\mathrm{pH}(5.0-6.4)$ similar to the trend observed for the variation of redox potential with $\mathrm{pH}$. Given that tannic acid is a weak acid with a pKa value of 5.12 (33), these observations suggest that ionised tannin molecules aid in the reduction of chloroauric acid and they also act as the charge determining ions for stabilisation. The estimated trend in the degree of dissociation is found to correlate with reaction mixture $\mathrm{pH}$ and not with the initial $\mathrm{pH}$ of tannic acid solution. This is plausible as tannin molecules are charged by dissociation of phenolic $\mathrm{H}+$ ions, and this process responds rapidly to changes in environmental $\mathrm{pH}$ brought about by mixing.

Overall, these results show that gold nanoparticle size distribution is determined by a fine balance between the reactivity of precursors and coalescence in the initial period, which can be manipulated by controlling the initial $\mathrm{pH}$ of reactants and reaction mixture $\mathrm{pH}$ respectively. Figure 4 is a schematic illustration of the various pathways described earlier. An important

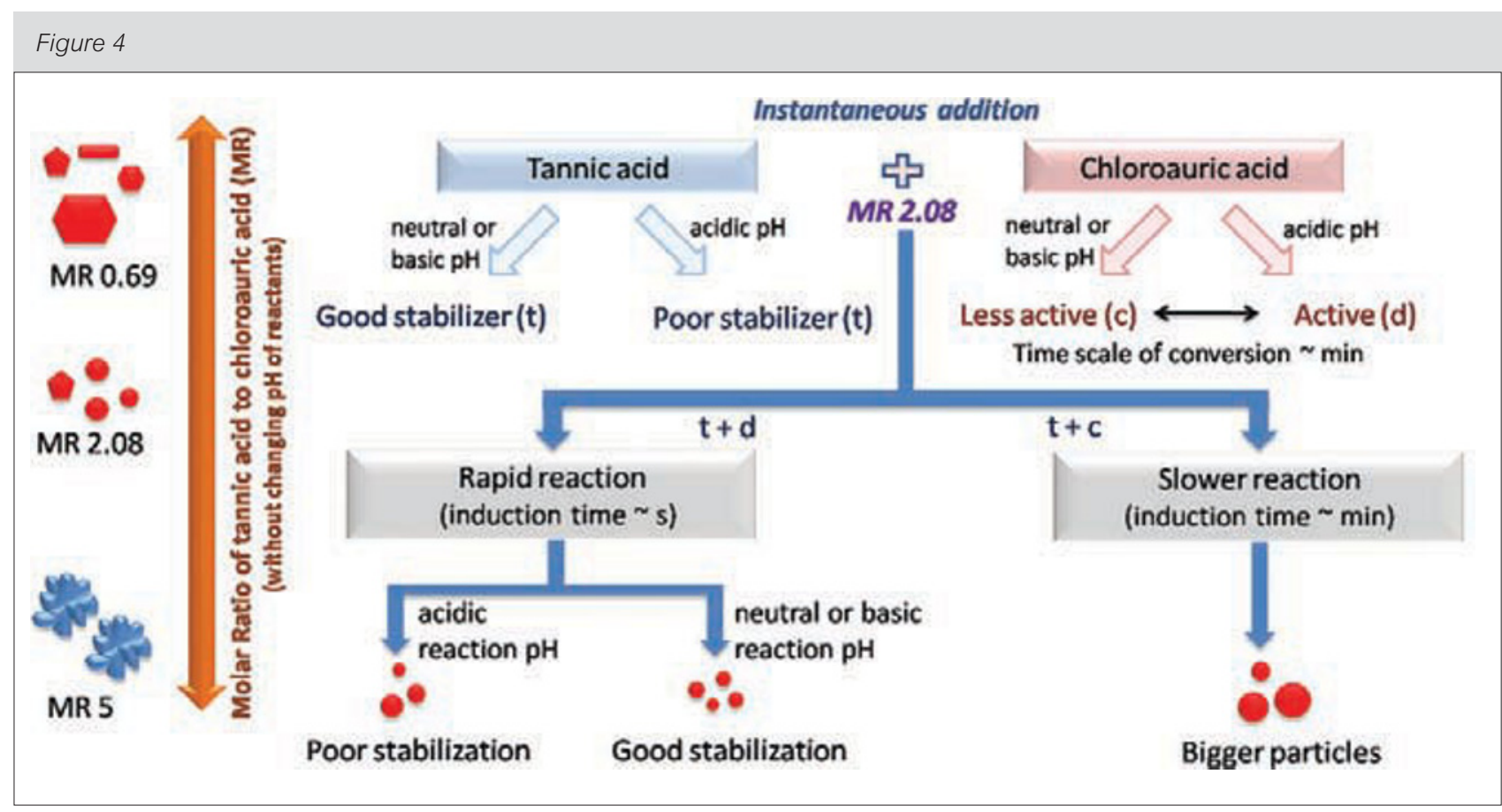

Schematic summary of the effect of MR and the roles of reagent and reaction mixture $\mathrm{pH}$ in determining nanoparticle size and shape 
outcome is that independent control over reactivity and stabilisation can be achieved by manipulating the $\mathrm{pH}$ of the precursor solutions.

\subsection{Effect of varying addition protocols}

As the objective is to achieve size-control, we focused our attention on experiment 'B', which exhibited the desirable characteristics of rapid nucleation and high stability (Note: experiment ' $F$ ' was not considered because of the need to add both an acid and a base for adjusting $\mathrm{pH}$, which can lead to large variations in ionic strength and affect colloidal stability). In experiment 'B', tannic acid was added as one portion into chloroauric acid solution and so, during the initial period of mixing (mixing timescale $\sim 2$ s) an acidic environment was present around the growing nanoparticles possibly leading to coalescence.
Hence, an experiment ('G') was carried out to minimize coalescence during mixing by altering the order of addition such that the growing nanoparticles were typically in alkaline medium. This was achieved by simply reversing the order of addition (i.e. chloroauric acid into tannic acid), while maintaining the initial $\mathrm{pH}$ of reagents, overall amount of reagents, the volume of the added reagent, and the volume of the total reaction mixture at values used in experiment 'B'. For comparison, a $1 \mathrm{~mL}$ sample was prepared by rapidly mixing ( $\sim$ in $3 \mathrm{~ms}$ ) the two reagents in SFM (experiment $\mathrm{H}$ ), while maintaining the concentration, initial $\mathrm{pH}$ and volumetric ratio similar to experiment 'B'.

Figures 5a and 5b show representative TEM images of nanoparticles formed in experiments ' $G$ ' and ' $H$ ', and their corresponding size distributions are

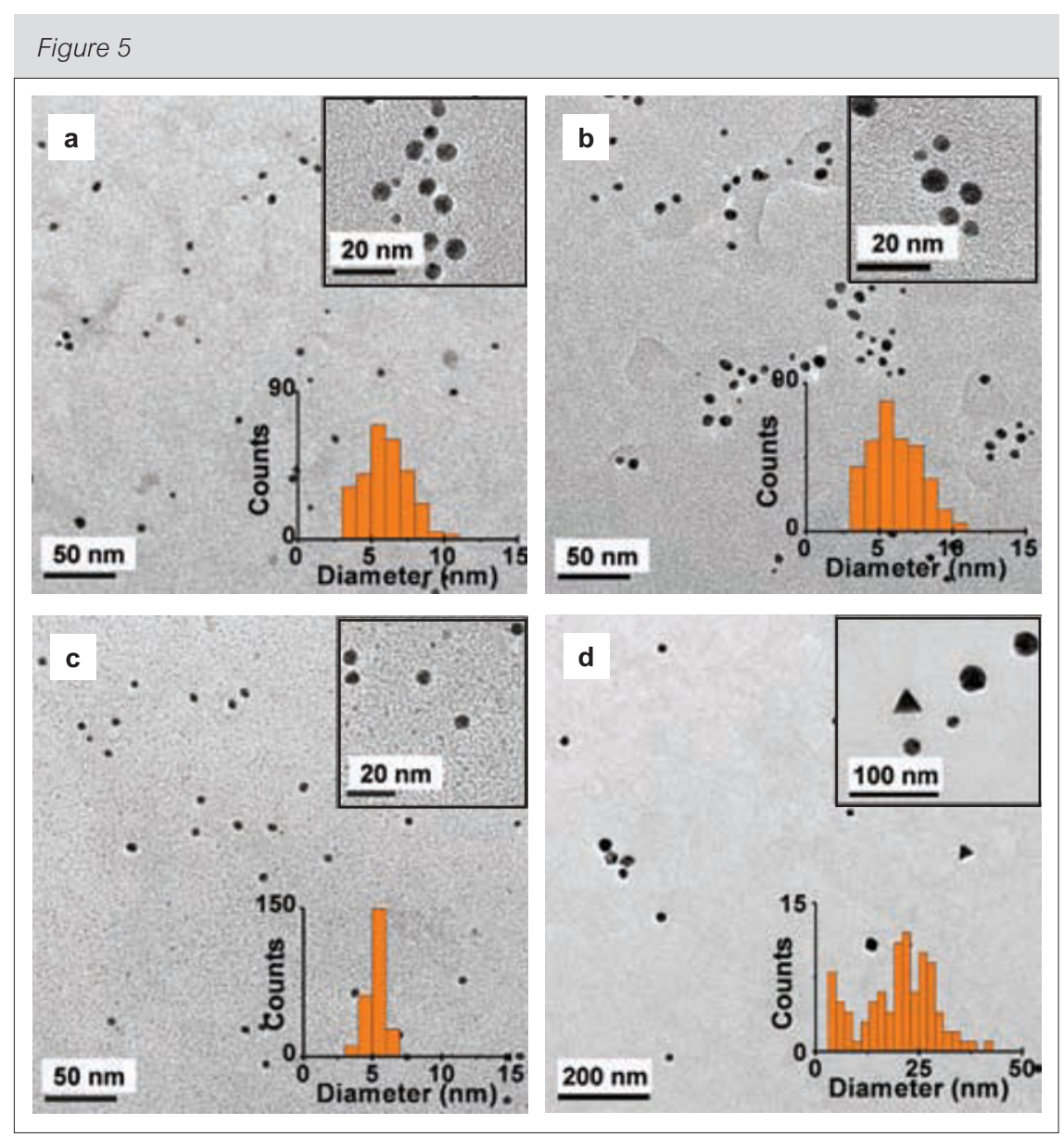

Representative TEM images highlighting the effect of order of addition, and rate of addition. Insets show higher magnification images and size distribution histograms. (a) Chloroauric acid added as one portion into tannic acid. Nanoparticle diameter $(\mu \pm \sigma)$ $=5.9 \pm 1.6 \mathrm{~nm}$. (b) Chloroauric acid and tannic acid were rapidly mixed using SFM. Nanoparticle diameter $(\mu \pm \sigma)=6.2 \pm 1.9 \mathrm{~nm}$. (c) Chloroauric acid added slowly $(1 \mathrm{~mL} /$ $\mathrm{min})$ into tannic acid. Nanoparticle diameter $(\mu \pm \sigma)=5.3 \pm 0.7 \mathrm{~nm}$. (d) Tannic acid added slowly (1 $\mathrm{mL} / \mathrm{min}$ ) into chloroauric acid. Nanoparticle diameter $(\mu \pm \sigma)=15.8 \pm 11.6 \mathrm{~nm}$ 
determined to be $5.9 \pm 1.6 \mathrm{~nm}$ and $6.2 \pm 1.9 \mathrm{~nm}$ respectively. The observed mean sizes follow the expected trend. The effect on standard deviation is however minimal, even in the case of rapid mixing, indicating that stabilisation of nanoparticles by adsorption of tannin requires a finite time during which particles may coalesce. Attempts to ameliorate this situation by using an excess of tannic acid did not yield the desired result. So, the next best strategy was to add diluted chloroauric acid slowly into tannic acid to result in an optimal particle number density, which would suppress coalescence in the initial period and provide adequate time for adsorption of tannin. Experiment 'l' was conducted by adding chloroauric acid at a rate of $\sim 1 \mathrm{~mL} / \mathrm{min}$ into a pool of tannic acid, while maintaining overall concentration and total volume of reaction mixture constant as in experiment ' $G$ ' (see methods for further details). For comparison, experiment 'J' was conducted by adding tannic acid slowly into a pool of chloroauric acid, keeping volume added, overall amounts and total volume same as in experiment 'G' and 'I'.

Figures 5c and 5d show representative TEM images of nanoparticles formed in experiments 'l' and 'J'; their corresponding size distributions were determined to be $5.3 \pm 0.7 \mathrm{~nm}$ and $15.8 \pm 11.6 \mathrm{~nm}$ respectively. The narrow standard deviation observed in experiment 'l' confirms that it is the dynamics of tannin adsorption that controls coalescence in the initial period. Considering that the extent of the mean size is smaller than in experiment ' $G$ ' for the same amount of gold atoms used, it is clear that the nanoparticle number density obtained ( $\sim 6.4 \times 10^{13}$ particles $\left./ \mathrm{mL}\right)$ is higher than those obtained using the fast addition protocol. Carrying out slow (dropwise) addition of the same amount of chloroauric acid into tannic acid at different concentrations (i.e. different MR values)

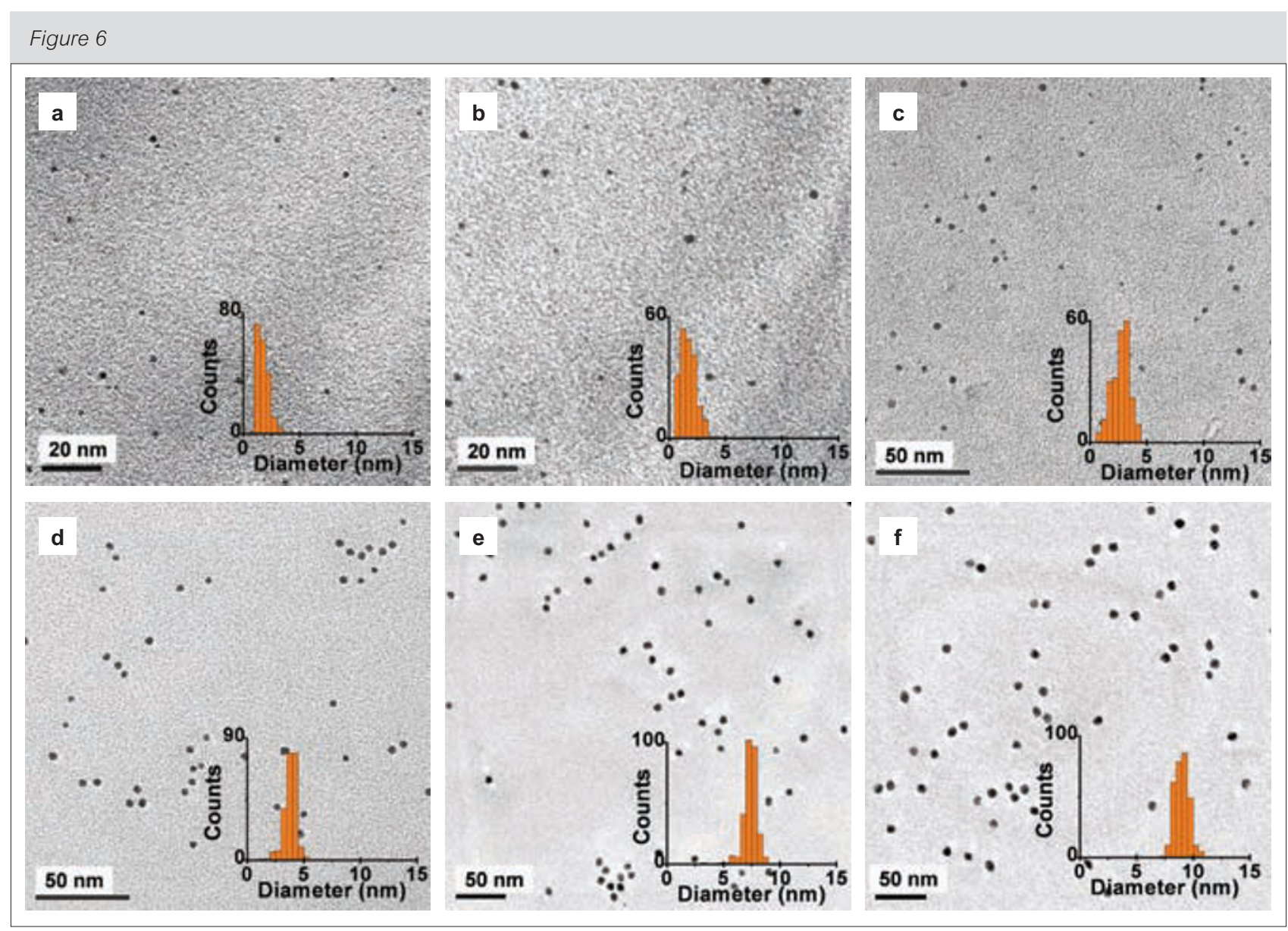

Representative TEM image of size-controlled nanoparticles formed by slow (dropwise) addition protocol. Insets show size distribution histograms. Nanoparticles were synthesized by slow ( 1mL/min) addition of (a) $0.2 \mathrm{~mL}$, (b) $0.4 \mathrm{~mL}$, (c) $2 \mathrm{~mL}$, (d) $5 \mathrm{~mL}$, (e) $40 \mathrm{~mL}$, of $0.64 \mathrm{mM}$ chloroauric acid into $15 \mathrm{ml}$ of $0.89 \mathrm{mM}$ tannic acid, while maintaining reaction mixture $\mathrm{pH}$ above 6.4. The nanoparticle size distributions $(\mu \pm \sigma$ ) are (a) $1.7 \pm 0.5 \mathrm{~nm}$, (b) $1.8 \pm 0.7$, (c) $2.7 \pm 0.8 \mathrm{~nm}$, (d) $3.8 \pm 0.6 \mathrm{~nm}$, (e) $7.4 \pm 0.6 \mathrm{~nm}$. In (f) nanoparticles were synthesized by slow (dropwise) addition of $10 \mathrm{~mL}$ of $0.32 \mathrm{mM}$ chloroauric acid solution into $15 \mathrm{~mL}$ of diluted gold colloid formed in experiment ' $l$ '. Nanoparticle diameter $(\mu \pm \sigma)=9.1 \pm 0.7 \mathrm{~nm}$ 
also resulted in quasi-spherical particles with lesser standard deviation as compared to the instantaneous addition protocol. In the case of experiment ' $\mathrm{J}$ ', the rather large size and polydispersity are attributed to the presence of nanoparticles with different shapes and morphology. The observed faceted nature of nanoparticles is in agreement with that reported for nanoparticles growing in an environment of excess chloride at acidic $\mathrm{pH}$ (34).

\subsection{Size-control by slow addition}

Given that the slow (dropwise) addition method for a rapid precipitation reaction is akin to a seeded growth approach after an initial period, we conjectured that smaller sized nanoparticles would be formed by simply adding lesser amount of chloroauric acid to the mixture. Figures $6 a-6 d$ summarize the results of such a set of experiments wherein particles ranging from $1.7 \mathrm{~nm}$ to $3.8 \mathrm{~nm}$ have been synthesized at room temperature within a matter of minutes. The constant value of standard deviation estimates (0.6-0.8 nm), limited by errors associated with thresholding of TEM images for particle size analysis (35), indicates that the growth is surface reaction controlled (i.e. the growth rate is independent of particle size). This process can be easily extended to form sizecontrolled nanoparticles with larger mean size, if care is taken to ensure that the reaction mixture $\mathrm{pH}$ never falls below 6.4. Figure 6e shows one such result obtained after the addition of $4 x$ the amount of chloroauric acid used in experiment ' $l$ '. The nanoparticle size distribution is found to be $7.4 \pm 0.6$ $\mathrm{nm}$, which reaffirms the surface-reaction controlled nature of the growth mechanism. The processing time required to form size controlled nanoparticles in the range of 2-10 $\mathrm{nm}$ can be further reduced by maximizing the amount of chloroauric acid for a given volume and number density of seeds (too high a concentration leads to secondary nucleation). Figure $6 f$ shows particles with a size distribution of $9.1 \pm 0.7 \mathrm{~nm}$ that were formed within 10 minutes by optimizing the concentration of chloroauric acid for a seed density of $6.4 \times 10^{12}$ particles $/ \mathrm{mL}$.

A simple mass balance for seeded growth shows that increase in mean nanoparticle diameter must be proportional to $1 / 3$ rd power of the moles (equivalent to volume at constant temperature) of chloroauric acid added. Figure 7 shows a plot of the predicted and the observed mean diameters as a function of the $1 / 3^{\text {rd }}$ power of the volume of chloroauric acid added. The observed values (including a few replicate samples) are seen to agree very well with the predicted values, which is a clear indication that

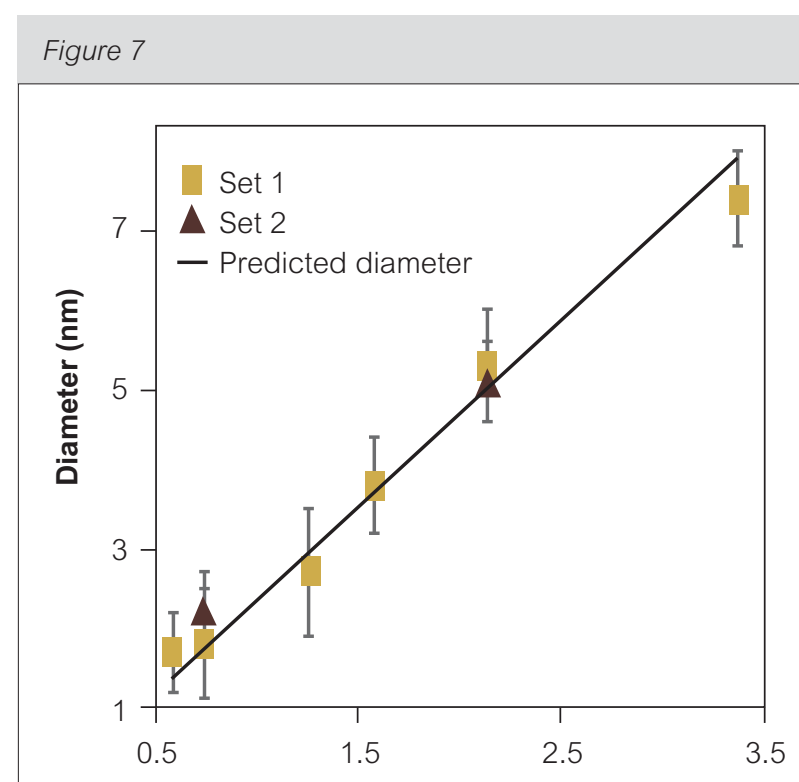

Volume of chloroauric acid added $\wedge 1 / 3(\mathrm{~cm})$

A plot of expected and observed nanoparticle diameter as a function of the amount of the 1/3rd power of the moles (equivalent to volume) of chloroauric acid added. The agreement between the predicted and observed diameters indicates that the slow (dropwise) addition process is akin to a one-shot nucleation-seeded growth process

the slow (dropwise) addition protocol is akin to oneshot process for seed formation followed by their controlled growth into nanoparticles.

\section{Conclusion}

In summary, the present work reports a significant advance in terms of the realisation of a simple, process capable of synthesising size-controlled gold nanoparticles rapidly under ambient conditions. The $\mathrm{pH}$ of precursor solutions, mode of contacting, and the dynamics of stabiliser adsorption vis-à-vis Brownian collision frequency are shown to be critical for tuning nanoparticle formation, growth, and coalescence. Thus, slower addition of chloroauric acid solution into tannic acid resulted in reduced polydispersity as compared to faster addition protocols. We have utilized such a slow (dropwise) addition protocol to synthesize size-controlled gold nanoparticles in the range of 2-10 nm within minutes at room temperature. The optimal process is shown to be similar to a one-shot nucleation-seeded growth technique and the growth mechanism is identified to be surface-reaction controlled under these conditions. These insights on independently manipulating reactivity and stabilisation can be extended to other redox reactions for rapidly 
synthesising size-controlled metal nanoparticles, as most systems exhibit $\mathrm{pH}$ dependent reactivity and stabilisation.

\section{Acknowledgement}

We thank Department of Science and Technology, New Delhi and the IISc-center for excellence in nanoelectronics (MIT-GOI) for funding. INI centre at IISc is acknowledged for TEM facilities. Prof. S.K. Biswas and Prof. S. Eswaramoorthyareacknowledged for their help with $\zeta$-potential measurements. We thank Prof. S. Sampath for help with measuring redox potentials. We thank Vijay Jain for help with AAS measurements. We acknowledge Internet Archive (www.archive.org) for access to historic texts.

\section{About the authors}

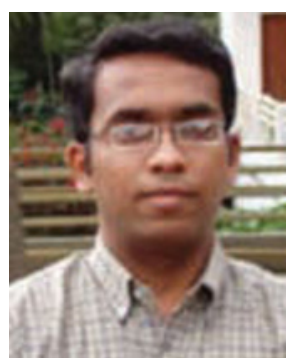

Sankar Kalidas Sivaraman is a research scholar in the Department of Chemical Engineering at IISc, Bangalore. His research focuses on the synthesis and ordering of gold nanoparticles for use in solar cells and chemical sensors.

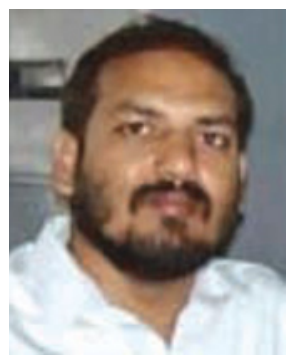

Sanjeev Kumar is an associate professor in the Department of Chemical Engineering at IISc, Bangalore. His research interests include modelling and experimental aspects of micellar processes, colloids and dispersions, and nanoparticle engineering.

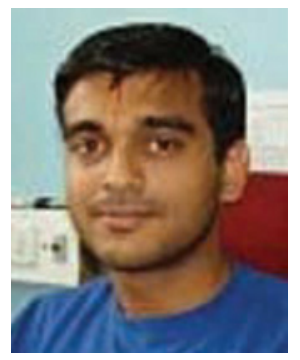

Venugopal Santhanam is an assistant professor in the Department of Chemical Engineering at IISc, Bangalore. His research interests are centered on the development of a process engineering 'toolkit' that will enable the use of nanoparticles as building blocks for a wide range of applications.

\section{References}

1 Ph. Buffat and J.P. Borel, Phys. Rev. A, 1976, 13, 2287

2 M.C. Daniel and D. Astruc, Chem. Rev., 2004, 104, 293

3 G. Peng, U. Tisch, O. Adams, M. Hakim, N. Shehada, Y.Y. Broza, S. Billan, R. Abdah-Bortnyak, A. Kuten and H. Haick, Nat. Nanotechnol, 2009, 4, 669

4 G.A. Somorjai and J.Y. Park, Top. Catal., 2008, 49, 126

5 J.A. Dieringer, A.D. McFarland, N.C. Shah, D.A. Stuart, A.V. Whitney, C.R. Yonzon, M.A. Young, X. Zhang and R.P. Van Duyne, Faraday Discussion, 2006, 132, 9

6 S. Kuhn, U. Hakanson, L. Rogobete and V. Sandoghdar, Phys. Rev. Lett., 2006, 97, 0174021

7 J.A. McLean, K.A. Stumpo and D.H. Russell, J. Am. Chem. Soc., 2005, 127, 5304

8 R.P. Andres, T. Bein, M. Dorogi, S. Feng, J.I. Henderson, C.P. Kubiak, W. Mahoney, R.G. Osifchin and R. Reifenberger, Science, 1996, 272, 1323

9 A.N. Shipway, E. Katz and I. Willner, ChemPhyChem, 2000, 1, 18

10 S.E. Skrabalak and Y. Xia, ACS Nano, 2009, 3, 10

11 J.E. Hutchinson, ACS Nano, 2008, 2, 395

12 H.B. Weiser, 'Inorganic Colloid Chemistry Vol.I: The Colloidal Elements', John Wiley and Sons: New York, 1933, pp.43

13 C.C. Wu and D.H. Chen, Gold Bull., 2007, 40, 206

14 V. Kumar and S.K. Yadav, J. Chem. Technol. Biotechnol., 2009, 84, 151

15 R.G. DiScipio, Anal. Biochem., 1996, 236, 168

16 I. Hussain, S. Graham, Z. Wang, B. Tan, D.C. Sherrington, S.P. Rannard, A.I. Cooper and M. Brust, J. Am. Chem. Soc., 2005, 127, 16398

17 S.K. Sivaraman, I. Elango, S. Kumar and V. Santhanam, Curr. Sci., 2009, 97, 1055

18 W. Ostwald, 'An Introduction to Theoretical and Applied Colloid Chemistry'John Wiley and Sons: New York, 1917, p.23

19 J.W. Slot and H.J. Geuze, Eur. J. Cell Biol., 1985, 38, 87

20 D.A. Handley, 'Methods for Synthesis of Colloidal Gold' in Colloidal Gold: Principles Methods and Applications, Vol I, Ed: M. A. Hayat, Academic Press: San Diego, 1989, p.18

21 J. Turkevich, P.C. Stevenson and J. Hiller, Discuss. Faraday Soc., 1951, 11, 55

22 J. Liu, F. He, T.M. Gunn, D. Zhao and C.B. Roberts, Langmuir, 2009, 25, 7116

23 S.M. Saraiva and J.F. Oliveira, J. Disper. Sci. Technol., 2002, 23, 837

24 D. Kim, S. Jeong and J. Moon, Nanotechnology, 2006, 17, 4019

25 W.L. McCabe, J.C. Smith and P. Harriott, 'Unit Operations of Chemical Engineering, 5th edition' McGraw-Hill Book Co: Singapore, 1993, p.242 
26 L.L. Ma, M.D. Feldman, J.M. Tam, A.S. Paranjape, K.K. Cheruku, T.A. Larson, J.O. Tam, D.R. Ingram, V. Paramita, J.W. Villard, J.T. Jenkins, T. Wang, G.D. Clarke, R. Asmis, K. Sokolov, B. Chandrasekar, T.E. Milner and K.P. Johnston, ACS Nano, 2009, 3, 2686

27 J.J. Beaver and R.H. Muller, J. Am. Chem. Soc., 1928, 50, 304

28 D.V. Goia and E. Matijevic, Colloids. Surf. A: Phys. Eng. Aspects, 1999, 146, 139

29 J. Xi, X. Song, J. Li, Y. Bai, W. Yang and X. Peng, J. Am. Chem. Soc., 2007, 129, 13939
30 W. Patungwasa and J.H. Hodak, Mater. Chem. Phys., 2008, 108, 45

31 A. Majzik, R. Patakfalvi, V. Hornok and I. Dékány, Gold Bull., 2009, 42, 113

32 F.H. Fry, G.A. Hamilton and J. Turkevich, Inorg. Chem., 1966, 5, 1943

33 J.H. An and S. Dultz, Appl. Clay Sci. 2007, 36, 256

34 M. Tsuji, N. Miyamae, M. Hashimoto, M. Nishio, S. Hikino, N. Ishigami and I. Tanaka, Colloids. Surf. A: Phys. Eng. Aspects, 2007, 302, 587

35 W.D. Pyrz and D.J. Buttrey, Langmuir, 2008, 24, 11350 